\title{
Perfil bacteriano das superfícies e equipamentos do Bloco Cirúrgico de um Hospital Universitário
}

\author{
Angela Bastos dos Santos ${ }^{a^{*}}$, Katia Suely Batista Silva ${ }^{a}$, Mirthes Maria Rodrigues \\ Santana $^{\mathrm{a}}$, Marilia Wortmann Marques ${ }^{\mathrm{b}}$, Carine Rosa Naue ${ }^{\mathrm{a}}$
}

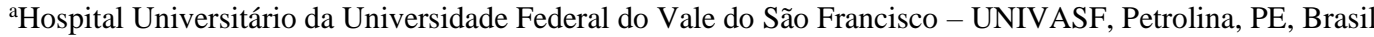

${ }^{\mathrm{b}}$ Instituto Federal Farroupilha - IFFAR, Panambi, RS, Brasil

\section{Histórico do Artigo: Recebido em: 25/02/2020 \\ Aceito em: \\ $23 / 04 / 2020$}

Palavras chave: Equipamentos; bactérias; infecção hospitalar

Keywords:

Equipment; bacteria; hospital infection.

\section{RESUMO}

As superfícies em um ambiente hospitalar contribuem para a contaminação cruzada secundária. Tal contaminação acontece através do contato das mãos dos profissionais com a manipulação dos dispositivos ou equipamentos e as suas superfícies, que poderão estar contaminadas e acabar contaminando os pacientes. É importante ressaltar que o comportamento dos profissionais de saúde tem grande influência de maneira direta no que tange a transmissão de infecção hospitalar. $\mathrm{O}$ presente estudo objetivou analisar o perfil bacteriano de superfícies e equipamentos do Bloco Cirúrgico de um Hospital Universitário. Trata-se de um estudo transversal e descritivo onde foram amostradas 06 salas do Bloco Cirúrgico e analisadas as seguintes superfícies e equipamentos: braçadeira, mesa cirúrgica, monitor multiparamétrico, oxímetro, foco de luz e carrinho do anestesista. As amostras foram coletadas, utilizando-se um molde de área de $1 \mathrm{~cm}^{2}$ e swabs embebidos em solução salina. O total de bactérias encontradas foi de 125, destas 5 são consideradas bactérias possíveis causadoras de infecções hospitalares, a saber: Acinetobacter baumannii (3 isolados) e Enterobacter sp. (2 isolados). Com relação a resistência, o Acinetobacter baumannii foi $100 \%$ resistente aos carbapenêmicos, cefalosporinas e quinolonas. Já os isolados de Enterobacter $s p$. foram $100 \%$ resistente a ampicilina e $100 \%$ sensível a sulfametoxazol + trimetropima, cefepima, meropenem, ciprofloxacino, amicacina, ceftriaxona, ampicilina + sulbactam, tetraciclina, cloranfenicol, piperacilina + tazobactam e aztreonam. As superfícies dos equipamentos do Bloco Cirúrgico apresentaram possíveis bactérias causadoras de infecções hospitalares demonstrando elevada resistência aos antibióticos.

\section{Bacterial profile of Surgical Block surfaces and equipment of a University Hospital} \begin{abstract}
Surfaces in a hospital environment contribute to secondary cross-contamination. Such contamination happens through the contact of the professionals' hands with the manipulation of devices or equipment and their surfaces, which may be contaminated and end up contaminating patients. It is important to emphasize that the behavior of health professionals has a great direct influence on what the transmission of hospital infection. The present study aimed to analyze the bacterial profile of surfaces and equipment in the operating room of a university hospital. It is a cross-sectional and descriptive study in which 06 rooms in the operating room were sampled and the following surfaces and equipment were analyzed: cuff, operating table, multiparametric monitor, oximeter, spotlight and anesthetist's cart. The samples were collected, using a $1 \mathrm{~cm}^{2}$ area mold and swabs soaked in saline solution. The total number of bacteria found was 125, of which 5 are considered possible bacteria that cause hospital infections, namely: Acinetobacter baumannii ( 3 isolates) and Enterobacter sp. (2 isolated). Regarding resistance, Acinetobacter baumannii was $100 \%$ resistant to carbapenems, cephalosporins and quinolones. The Enterobacter $s p$. were $100 \%$ resistant to ampicillin and $100 \%$ sensitive to sulfamethoxazole + trimethoprim, cefepime, meropenem, ciprofloxacin, amikacin, ceftriaxone, ampicillin + sulbactam, tetracycline, chloramphenicol, piperacillin + tazobactam and aztreonam. The surfaces and equipment of the operating room showed possible bacteria that caused hospital infections with high resistance to antibiotics.
\end{abstract}

\section{Introdução}

A infecção hospitalar (IH) é definida como toda infecção que é adquirida após 72 horas

\footnotetext{
*Autor correspondente: angelabast61@gmail.com (Santos A.B.)
} 
da admissão no serviço hospitalar e que se apresenta durante o período de internação ou após a alta, desde que seja capaz de relacioná-la com o tempo de internação, ou ainda aos procedimentos realizados no âmbito hospitalar (1).

Sabe-se que o comportamento dos profissionais de saúde tem grande influência de maneira direta no que tange a transmissão da $\mathrm{IH}$, visto que a realização das práticas de forma incorreta irá atuar como um meio de transmissão de micro-organismos patogênicos aos pacientes (2).

Já com relação as superfícies, elas mostram-se como um baixo risco de transmissão direta de infecção, porém, contribuem para a contaminação cruzada secundária. Essa contaminação se dá através do contato das mãos dos profissionais de saúde com a manipulação dos dispositivos ou equipamentos e as suas superfícies, que poderão ser contaminadas e por fim terminar contaminando os pacientes e outros locais que são manipulados pelos profissionais de saúde com frequência (3).

Em relação ao ambiente, estão incluídas todas as superfícies hospitalares, pois apesar de levar a um risco mínimo de transmissão direta de infecção, pesquisas têm revelado sua importância em relação à multiplicação de micro-organismos, visto que contribuem para a infecção cruzada, por intermédio dos profissionais de saúde, procedendo como fonte de dispersão de patógenos responsáveis pelas infecções relacionadas a saúde (4). A infecção hospitalar é de grande importância, em virtude de sua frequência e alta morbimortalidade (5).

Diante da importância do ambiente como possível reservatório e fonte de transmissão de micro-organismos que causam IH, o presente estudo objetivou analisar o perfil bacteriano de superfícies e equipamentos do Bloco Cirúrgico de um Hospital Universitário.

\section{Materiais e métodos}

O experimento foi realizado no Bloco Cirúrgico de um Hospital Universitário do Sertão do Vale do São Francisco do Estado de Pernambuco.

Trata-se de um estudo transversal e descritivo desenvolvido com o intuito de determinar o papel das superfícies e equipamentos como possíveis reservatórios de agentes etiológicos bacterianos associados às infecções hospitalares.

No Bloco Cirúrgico foram amostradas as salas destinadas as seguintes especialidades: sala 1: cirurgia buco-maxilo-facial, sala 2: escleroterapia; sala 3: cirurgia ortopédica; sala 4: cirurgia geral; sala 5: cirurgia vascular e sala 6: cirurgia geral. Em cada sala foram analisadas as seguintes superfícies e equipamentos: braçadeira, mesa cirúrgica, monitor multiparamétrico, oxímetro, foco de luz e carrinho do anestesista.

As amostras das superfícies e dos equipamentos foram coletadas, utilizando-se um molde de papel filtro de área de $1 \mathrm{~cm}^{2}$ e swabs embebidos em solução salina. O molde de papel filtro de $1 \mathrm{~cm}^{2}$ foi utilizado com o objetivo de padronização das áreas amostradas. Após a passagem do $s w a b$, nas superfícies e equipamentos, os mesmos foram armazenados em tubo contendo $5 \mathrm{~mL}$ meio liquido BHI (Brain Heart Infusion). Em seguida, as amostras foram transportadas para o Laboratório de Análises Clínicas/ Setor Microbiologia do Hospital UniversitárioUNIVASF (EBSERH), onde foram realizadas as análises microbiológicas. Cada superfície e equipamento foram amostrados em 4 pontos diferentes.

No laboratório, os caldos BHI foram incubados a $37^{\circ} \mathrm{C}$ por 48 horas. Para o isolamento das bactérias, as amostras foram semeadas em Ágar Sangue (AS) e incubadas a $37^{\circ} \mathrm{C}$ por 24 horas. Após o período de incubação, nas placas que apresentaram crescimento, foi feito o repique para isolamento das colônias puras e posteriormente realizado a coloração de gram e as provas bioquímicas para a identificação de cada espécie. Para identificação dos cocos Gram-positivos foi realizada a prova de catalase. Quando a catalase foi positiva, foi realizada a prova de coagulase, e quando a catalase foi negativa, utilizou-se o Kit para 
Enterococcus, bacitracina e optoquina para diferenciar o gênero Enterococcus das espécies de Streptococcus. Para a identificação de bacilos gram negativos foram utilizados os kits para identificação de Enterobactérias e/ou de Não Fermentadores de glicose PROBAC®, conforme as instruções do fabricante.

As bactérias identificadas foram submetidas ao antibiograma pelo método de Ágar Difusão em Disco conforme as instruções CLSI (2018) e a escolha dos antimicrobianos realizada conforme o micro-organismo isolado. Os resultados foram armazenados e analisados em uma planilha eletrônica de dados (Microsoft Excel® 2003).

A pesquisa foi conduzida dentro dos padrões éticos, pois a Instituição autorizou a pesquisa através da emissão da carta de anuência.

\section{Resultados}

O total de bactérias encontradas, independente das salas, equipamentos e superfícies amostradas foi de 125, destas 5 (4\%) são consideradas possíveis bactérias causadoras de infecções hospitalares, a saber: 3 isolados de Acinetobacter baumannii, 2 de Enterobacter sp. (Figura 1). O restante das bactérias isoladas encontra-se no grupo dos Staphylococcus coagulase negativa ( 87 isolados), que em sua grande parte são colonizadoras da pele, mas dependendo da situação imunológica do paciente, esses micro-organismos podem tornarse um patógeno e ser um fator a mais para o aumento de risco de infecção e as bactérias do ambiente (33 isolados), assim chamadas no presente estudo, pois não foram identificadas pelas provas bioquímicas utilizadas na rotina de identificação de bactérias de interesse clínico e possuíam morfologia de bactérias presentes no ambiente, geralmente bacilos gram positivo (Figura 1).

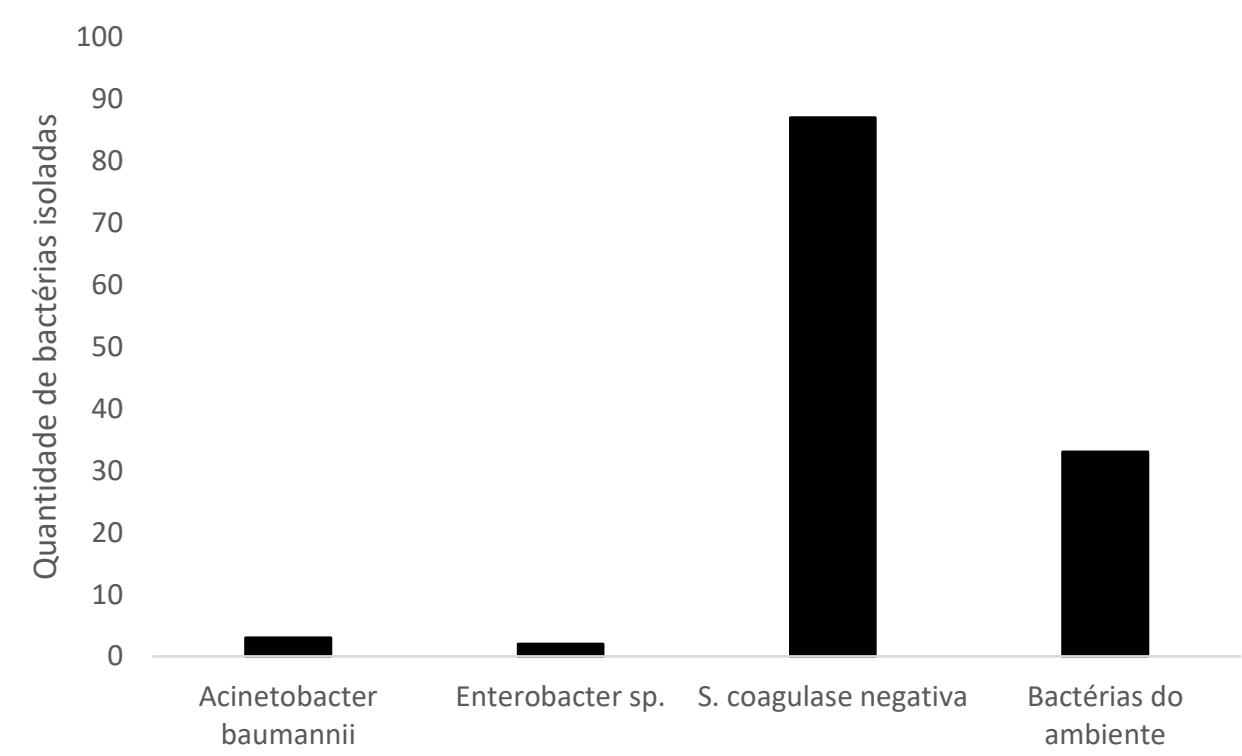

Figura 1 - Quantidade de bactérias isoladas em superfícies e equipamentos do Bloco Cirúrgico.

Observa-se na Tabela 1 que em todos os equipamentos e superfícies analisados foram encontrados isolados bacterianos que podem ou não causar infecções hospitalares. Na sala 1 (cirurgia buco-maxilo-facial) a maioria das superfícies dos equipamentos foram colonizadas por Staphylococcus coagulase negativa, como por exemplo: Braçadeira, mesa cirúrgica, oxímetro, mesa multiparamétrica e carrinho do anestesista.

Com relação a sala 2 (escleroterapia), houve uma maior ocorrência de micro-organismos 
do tipo Staphylococcus coagulase negativa e bactéria do ambiente. Os equipamentos onde tiveram a ocorrência destes dois gêneros foram: Mesa cirúrgica, mesa multiparamétrica e foco de luz. No restante das superfícies e equipamentos foram isolados apenas Staphylococcus coagulase negativa.

Já na sala 3 (cirurgia ortopédica) observou-se que as bactérias do ambiente e Staphylococcus coagulase negativa estiveram presentes na maioria dos equipamentos, exceto na mesa multiparamétrica, que foi observado Enterobacter sp., sendo uma bactéria possível causadora de infecções hospitalares.

Verificou-se também que na sala 4 (cirurgia geral) houve um maior número de bactérias residentes da microbiota da pele, o Staphylococcus coagulase negativa, presente nas superfícies, ao passo que na sala 5 (cirurgia vascular) notou-se que a maioria dos equipamentos não houve crescimento bacteriano, com exceção da mesa cirúrgica, onde foi isolado o A. baumannii, que é uma bactéria possível causadora de infecções hospitalares. Na sala 6 houve, em sua maior parte, bactérias presentes nas superfícies do tipo. Staphylococcus coagulase negativa.

É preciso ressaltar que tanto a mesa multiparamétrica, quanto a mesa cirúrgica são equipamentos, onde o paciente encontra-se em contato direto durante todo $o$ procedimento cirúrgico, e que é uma superfície manuseada por toda equipe no decorrer da intervenção cirúrgica, o que poderá ocasionar infecções cruzadas.

Tabela 1 - Espécies bacterianas isoladas nas superfícies e equipamentos das diferentes salas amostradas do Bloco Cirúrgico de um Hospital Universitário

\begin{tabular}{|c|c|c|}
\hline LEITOS & Equipamentos e superfícies & \\
\hline \multirow[t]{7}{*}{ SALA 1} & Braçadeira & Staphylococcus coagulase negativa \\
\hline & Mesa cirúrgica & Staphylococcus coagulase negativa \\
\hline & Mesa multiparamétrica & Staphylococcus coagulase negativa e bactéria do ambiente \\
\hline & Mesa grande & Não houve crescimento bacteriano \\
\hline & Oxímetro & Staphylococcus coagulase negativa \\
\hline & Foco de luz & Bactéria do ambiente \\
\hline & Carrinho do anestesista & Staphylococcus coagulase negativa \\
\hline \multirow[t]{7}{*}{ SALA 2} & Braçadeira & Staphylococcus coagulase negativa \\
\hline & Mesa cirúrgica & Staphylococcus coagulase negativa e bactéria do ambiente \\
\hline & Mesa multiparamétrica & Staphylococcus coagulase negativa e bactéria do ambiente \\
\hline & Mesa grande & Staphylococcus coagulase negativa \\
\hline & Oxímetro & Staphylococcus coagulase negativa \\
\hline & Foco de luz & Staphylococcus coagulase negativa e bactéria do ambiente \\
\hline & Carrinho do anestesista & Staphylococcus coagulase negativa \\
\hline \multirow[t]{7}{*}{ SALA 3} & Braçadeira & Staphylococcus coagulase negativa \\
\hline & Mesa cirúrgica & Bactéria do ambiente \\
\hline & Mesa multiparamétrica & Enterobacter sp. \\
\hline & Mesa grande & Bactéria do ambiente \\
\hline & Oxímetro & Staphylococcus coagulase negativa \\
\hline & Foco de luz & Bactéria do ambiente \\
\hline & Carrinho do anestesista & Staphylococcus coagulase negativa e bactéria do ambiente \\
\hline \multirow[t]{7}{*}{ SALA 4} & Braçadeira & Staphylococcus coagulase negativa \\
\hline & Mesa cirúrgica & Staphylococcus coagulase negativa \\
\hline & Mesa multiparamétrica & Bactéria do ambiente \\
\hline & Mesa grande & Staphylococcus coagulase negativa \\
\hline & Oxímetro & Staphylococcus coagulase negativa \\
\hline & Foco de luz & Staphylococcus coagulase negativa \\
\hline & Carrinho do anestesista & Bactéria do ambiente \\
\hline \multirow[t]{7}{*}{ SALA 5} & Braçadeira & Staphylococcus coagulase negativa \\
\hline & Mesa cirúrgica & Acinetobacterbaumannii \\
\hline & Mesa multiparamétrica & Staphylococcus coagulase negativa e bactéria do ambiente \\
\hline & Mesa grande & Não houve crescimento bacteriano \\
\hline & Oxímetro & Não houve crescimento bacteriano \\
\hline & Foco de luz & Não houve crescimento bacteriano \\
\hline & Carrinho do anestesista & Staphylococcus coagulase negativa \\
\hline \multirow[t]{7}{*}{ SALA 6} & Braçadeira & Bactéria do ambiente \\
\hline & Mesa cirúrgica & Staphylococcus coagulase negativa \\
\hline & Mesa multiparamétrica & Bactéria do ambiente \\
\hline & Mesa grande & Staphylococcus coagulase negativa \\
\hline & Oxímetro & Staphylococcus coagulase negativa e bactéria do ambiente \\
\hline & Foco de luz & Staphylococcus coagulase negativa \\
\hline & Carrinho do anestesista & Staphylococcus coagulase negativa \\
\hline
\end{tabular}


Em relação ao perfil de resistência aos antibióticos, os isolados de A. baumannii foram $100 \%$ resistentes a sulfametoxazol + trimetropima, cefepima, meropenem, ciprofloxacino, amicacina, ceftriaxona, 33,33\% resistentes a ampicilina + sulbactam e $100 \%$ intermediário a gentamicina. Já os isolados de Enterobacter sp. foram $100 \%$ resistentes a ampicilina e 100\% sensíveis a sulfametoxazol + trimetropima, cefepima, meropenem, ciprofloxacino, amicacina, ceftriaxona, ampicilina + sulbactam, tetracilina, cloranfenicol, piperacilina + tazobactam e aztreonam.

\section{Discussão}

Pesquisas realizadas neste mesmo sentido mostram uma microbiota muito parecida com a encontrada no presente trabalho. Dresch et al. (3) realizaram o isolamento de bactérias das seguintes superfícies: Trilho da cama, mesa de cabeceira, dispensador de álcool, válvulas intravenosas, cadeiras de cabeceira, carrinhos de equipamentos, bombas de infusão, estetoscópios, monitores multiparamétricos, carrinhos de emergência, maçanetas e teclados, entre outros. Nas superfícies, as principais bactérias isoladas, pelos pesquisadores foram: Staphylococcus aureus, Staphylococcus coagulase negativa (SCN), Pseudomonas aeruginosa, Klebsiella pneumoniae e A. baumannii.

Os Staplylococcus coagulase negativa são bactérias gram-positivas tidas como imóveis. Atualmente são conhecidas por formarem colônias comensais nas mucosas ou tecido cutâneo, ocorrendo tanto em animais, quanto em seres humanos. Os micro-organismos relacionados aos seres humanos são: Staplylococcus epidermidis, Staplylococcus haemolyticus, Staplylococcus capitis, Staplylococcus hominis, Staplylococcus pettenkoferi, Staplylococcus simulans, Staplylococcus warneri, Staplylococcus saprophyticus, Staplylococcus lugdunensis, entre outros. São bactérias que manifestam predisposição por sítios nutritivos, úmidos e de fácil colonização. Tais bactérias estão associados à criação de biofilme e sua colonização está comparada à forma de apropriação no tecido do hospedeiro, agressão, adesão, insistência da imunidade inata e da imunidade adaptativa e a interação hospedeira-bactéria (6). A elevada ocorrência deste grupo de bactérias neste presente trabalho se deve ao fato destas bactérias serem colonizadoras da pele e as superfícies e equipamentos serem frequientemente tocados pelos profissionais de saúde.

A outra bactéria encontrada no presente trabalho foi A. baumannii. Essa bactéria é caracterizada como um cocobacilo gram negativo, que nos últimos anos tem sido apresentado como um importante patógeno nosocomial. As infecções obtidas durante o período de internamento hospitalar e que são causadas por $A$. baumannii, em geral, abrangem principalmente o trato respiratório, urinário e feridas, sendo capaz de progredir para uma septicemia (7). Esse gênero bacteriano, tem predisposição por superfícies secas e sua sobrevida nesses ambientes é maior que 25 dias, o que justifica sua maior patogenicidade em pacientes que encontram-se hospitalizados (8).

Essa espécie bacteriana apresenta vários mecanismos que as tornam uma ameaça em Instituições de Saúde, como a sua habilidade de produzir biofilmes e sobreviver à dessecação em superfícies abióticas, como por exemplo os dispositivos médicos e superfícies ambientais, a facilidade de aderir, colonizar e penetrar nas células epiteliais dos indivíduos, estrutura que contribui para a resistência a antibióticos, e por fim, sua tendência para adquirir material genético estrangeiro por meio da transferência lateral de genes e favorecer sua própria sobrevivência sob a pressão de antibióticos e seleção de hospedeiros (8).

O A. baumannii apresenta diferentes mecanismos envolvidos com o processo de resistência aos antibióticos. O mais frequentes são aos antibióticos b-lactâmicos, que pode 
ocorrer pela produção de b-lactamases, baixa permeabilidade da membrana externa, ausência de porinas, modificações nos sítios de ligação dos antibióticos e hiperexpressão de bombas de efluxo (9).

Os antibióticos da classe dos carbapenêmicos executavam um papel considerado importante no manejo de infecções hospitalares, conhecidas como severas, causadas por esse patógeno. Contudo, o surgimento de cepas resistentes aos carbapenêmicos é cada vez mais constante devido à produção de enzimas beta-lactamases pertencentes às classes $\mathrm{D}$ e $\mathrm{B}$ de Ambler, as metalo-beta-lactamases (MBLs) e a produção de carbapenemases do tipo OXA (10).

Em relação ao gênero Enterobacter, ele é um micro-organismo gram-negativo, anaeróbico facultativo, em forma de bastonete, não formador de esporos, pertencentes à família Enterobacteriaceae. Existem duas espécies que são conhecidas, a Enterobacter aerogenes e a Enterobacter cloacae, elas alcançaram relevância clínica como bactérias oportunistas e revelaram-se como patógenos nosocomiais de pacientes internados em unidade de terapia intensiva, em particular para aqueles que se encontram em ventilação mecânica (11).

Esse gênero bacteriano participa da microbiota intestinal normal dos indivíduos e animais e os locais que ela pode ser encontrada são: solo, esgoto, água e produtos alimentícios. Elas possuem alguns mecanismos genéticos que as tornam resistentes aos antimicrobianos e um dos principais mecanismos é a produção de beta-lactamases que são enzimas que inativam os antimicrobianos beta-lactâmicos (12). Segundo o mesmo autor, entender os mecanismos que estão envolvidos no processo de resistência aos antimicrobianos é de suma importância, tanto para uso adequado desses fármacos na prática clínica, como também para o desenvolvimento de novos antimicrobianos que possam quebrar a barreira de resistência bacteriana (12).

É importante salientar que biofilmes presentes em superfície seca, nos setores clínicos foram recentemente estudadas e percebeu a sobrevivência de bactérias vegetativas, que ficaram aderidas nas superfícies por longos períodos (13). Portanto sabe-se que a limpeza e desinfecção de forma correta, contribui de forma eficaz para a diminuição desses microorganismos e consequentemente para a diminuição da infecção cruzada, relacionada ao contato com superfícies.

\section{Conclusão}

O presente estudo mostra que possíveis bactérias causadoras de infecções hospitalares como o Acinetobacter baumannii e Enterobacter sp. com elevada resistência aos antimicrobianos estão presentes nas superfícies e equipamentos do Bloco Cirúrgico e consequentemente podem causar possíveis infecções cruzadas. Os profissionais que trabalham neste setor devem estar cientes deste fato e adotar melhores medidas de desinfecção das superfícies e equipamentos, o que diminuirá o risco de infecções cruzadas.

\section{Referências}

1. Michelin AF, Fonseca MRCC. Perfil epidemiológico das infecções hospitalares na unidade de terapia intensiva de um hospital terciário. Rev Nursing 2018; 21(236): 2037-2041.

2. Silva RE, Silva ACA, Freitas AL, Sá CC, Santos IL, Silva VRV, et al. Conhecimento de estudantes da área de saúde sobre o controle e prevenção de infecções hospitalares. Rev Bras cien saúde 2018; 22(2): 131-138.

3. Dresch F, Birkheuer CF, Rampel C, Maciel MJ. Contaminação de superfícies localizadas em unidades de terapia intensiva e salas de cirurgia: uma revisão sistemática da literatura. Rev de Epidemiol e Control Infec 2018; 8(1): 85-91. 
4. Simch B, Dresch F, Maciel MJ. Microbiological analysis of a hospital sterilized material center: identification and resistance to antibiotics. Rev cont e saúde 2018; 18:(35): 95-103.

5. Veloso JO, Cardoso JL, Neves LS, Borges LFA, Pires CH, Lamaro L, et al. Methicilli n -resistant and vancomycin - intermediate Staphylococcus aureus colonizing patients and intensive care uniten vironment: virulence profile and genetic variability. APMIS. 2019; 127(11):717-726.

6. Acquaviva R, D’Angeli F, Malfa GA, Ronsisvalle S, Garozzo A, Stivala A, et al. Antibacterial and anti-biofilm activities of walnut pellicle extract (Juglans regia L.) against coagulase-negative staphylococci. Natural Product Research, 2019: 1-6.

7. Mota FS, Oliveira HA, Souto RCF. Perfil e prevalência de resistência aos antimicrobianos de bactérias Gram-negativas isoladas de pacientes de uma unidade de terapia intensiva. Revista brasileira de Análises Clínicas 2018; 50(3): 270-7.

8. Borges RM, Nunes CP. Infecções por acinetobacter baumannii em unidades de terapia intensiva. Revista de Medicina de Família e Saúde Mental 2019; 1(2): 17-23.

9. Martins AF, Barth AL. Acinetobacter multirresistente: um desafio para a saúde pública. Scientia Medica 2013; 23(1): 56-62.

10. Ricas RV, Marques TC, Yamoto ACA. Perfil de resistência de Acinetobacter baumannii a antimicrobianos em um hospital universitário de Cuiabá MT. Infarma Ciências Farmacêuticas 2013;25(4): 178-181.

11. Regli AD, Pagès JM. Enterobacter aerogenes and Enterobacter cloacae; versatile bacterial pathogens confronting antibiotic treatment. Front Microbiol. 2015; 6:392.

12. Paula VG, Quintanilha LV, Silva FAC, Rocha HF, Santos FL. Enterobactérias produtoras de carbapenemase: prevenção da disseminação de superbactérias em UTI's. Ciências da Saúde 2016; 14(2): 175-185.

13. Casini B, Tuvo B, Cristina ML, Spagnolo NA, Totaro M, Baggiani A, et al. Evaluation of an Ultraviolet C (UVC) Light-Emitting Device for Disinfection of High Touch Surfaces in Hospital Critical Areas. Int. J. Environ. Res. Public Health 2019, 16: 3572. 